\title{
Construction of College English Data Resources and Change of Teacher Positioning Using QoS Constraints
}

\begin{abstract}
Aijie Hu
Wuhan Technical College of Communications, Wuhan, Hubei 430065, China

Correspondence should be addressed to Aijie Hu; hajtina@whtcc.edu.cn

Received 17 January 2022; Revised 7 February 2022; Accepted 8 February 2022; Published 28 February 2022

Academic Editor: Tongguang Ni

Copyright (c) 2022 Aijie Hu. This is an open access article distributed under the Creative Commons Attribution License, which permits unrestricted use, distribution, and reproduction in any medium, provided the original work is properly cited.

Cultivating students' autonomous learning abilities has become a research hotspot in CE (College English) reform, and the new $\mathrm{CE}$ autonomous learning teaching mode with students at the center also requires teachers to change traditional teaching ideas. The traditional teaching mode and teacher orientation have changed dramatically as a result of the new situation of $\mathrm{CE}$ teaching reform. The design and implementation of a CE data resource base based on a web database demonstrates that these technologies provide people with greater convenience and practicality. A GA (Genetic Algorithm) based on a QoS (Quality of Service) constraint is proposed on this basis. This algorithm can find a suitable resource node in the computing resources for each subtask that meets the constraint conditions and assign it to the corresponding task. Information-based education has become a powerful driving force for educational revolution, with computer technology and network information technology making English classroom teaching richer, more flexible in form, rich in content, and searchable in data.
\end{abstract}

\section{Introduction}

The traditional teaching model has been greatly impacted and challenged as $\mathrm{CE}$ teaching reform continues to progress. The reform entails gradually transitioning from a teacher-centered teaching mode of imparting language knowledge and skills to a student-centered teaching mode that emphasizes the development of students' practical language use ability and autonomy in learning. This necessitates a shift in teachers' responsibilities. CE teachers face an urgent problem: how to fully utilize students' initiative of autonomous learning and make better use of the autonomous learning mode to improve their English level. Outside of the classroom, students can learn English in a variety of ways, and the popularity of multimedia teaching in college classrooms has changed CE (College English) teachers' requirements and orientation $[1,2]$. The design and implementation of a web-based CE data resource database, on the other hand, can not only realize the educational era's development but also promote network technology's development.

Make full use of social resources and use the "bringing in" method [3]. Good resources should be kept in the teaching resource pool, while outdated and obsolete resources should be discarded. The construction of a professional teaching resource pool should follow the education and teaching philosophy of "putting students' needs first and teachers second" and give full consideration to the characteristics and benefits of teaching resource pools [4]. Text, pictures, animation, and other forms of traditional learning resources can no longer meet the learning needs of learners. When streaming media technology is used to create a large number of audio and video resources, the teaching effect becomes more vivid and vivid $[5,6]$. Teachers should play an important role in the learning process by guiding students' autonomous learning activities, assisting students in mastering the proper learning methods, adjusting their learning progress according to their current situation, and setting personalized learning goals. English teachers should also assist students in gathering a variety of learning materials, organizing and analyzing them on a regular basis, and finally assisting students in developing the ability to learn independently [7]. Multimedia web teaching is not only simple and quick, but can also improve teachers' teaching quality while also ensuring teachers' and students' access to 
resources is reliable. The use of multimedia network teaching can greatly aid teachers in improving teaching quality, allowing teachers to improve their own teaching level to some extent [8].

In the case of emphasizing multimedia teaching, instead of seriously studying the characteristics of multimedia teaching, just emphasize the convergence of forms, which leads to the phenomenon of full teaching in multimedia classroom. The teaching methods of some teachers are not standardized, and the main points are not highlighted. Teachers only demonstrate and read multimedia courseware in multimedia teaching so that students can copy their teaching materials. Multimedia instruction is informationrich, fast-paced, and ever-changing. Students believe that, in the process of multimedia teaching, they are unable to grasp key points and have a strong memory and that it is difficult to digest what is said in class and take notes. As a result, this paper develops and implements a web-based CE data resource database. The platform of the $\mathrm{CE}$ data resource database and the teaching resources in the CE data resource database are divided into two sections.

\section{Related Work}

According to $[9,10]$, despite the fact that the construction of digital resources has reached a pinnacle, the overall quality is not high, and quality resources are limited; particularly the supply and service based on digital resources are too rough and crazy. In the compulsory education stage, [11] proposes the creation of "synchronous courses for famous teachers," which provides primary and secondary school students with courses from all grades and disciplines in the form of online video, further enriching the supply of quality education resources. Reference [12] has created a multimedia education resource database system with significant communication value. Users of such a network platform of teaching resources can share all types of teaching materials without being constrained by time or geography, and it provides a convenient and quick platform for teaching. According to [13], an English curriculum requires students to cultivate their daily communicative activities in addition to having good language skills. According to [14], English teachers should not only teach students basic English skills, but also consider their psychological well-being and promote their physical and mental development. According to [15], the new teaching model can provide students with rich, vivid, and intuitive information, expand students' cognitive space, shorten students' cognitive process, create a communication bridge, and help students improve their language and cultural awareness.

People's network dreams are embodied in the cloud computing vision. To some extent, distributed computing and grid computing reflect the concept of cloud computing, that is, combining idle resources distributed across a network to provide powerful computing power [16]. Many researchers have tried to use the resource scheduling algorithm in a grid environment to see if resource scheduling in the cloud is possible. In a distributed environment such as a grid, for resource scheduling, [17] describes a task scheduling algorithm for grid computing based on GA (Genetic Algorithm), which aims to maximize resource utilization and throughput. Reference [18] examines existing cloud computing platform solutions and technologies, then designs and analyzes a job scheduling algorithm for virtualization platforms, and proposes a scheduling scheme based on the threshold dynamic scheduling model. In [19], in light of GA's flaws, an improved algorithm for optimizing scheduling is proposed, and GA with a double fitness function is considered to find nodes that reduce task scheduling's average and total times. The half-validity method, proposed in [20], is an improved method for maintaining the timing consistency of real-time data objects. The update period of real-time data objects is set to half the effective interval length of real-time data objects in this method. As a result, even in the worst-case scenario, this algorithm can guarantee the timing consistency of real-time data objects. To achieve better optimization of system resource use, it is also suggested in $[21,22]$ to delay the sampling time of update transactions as long as possible and use a dynamic periodic transaction deadline.

\section{Method}

3.1. Construction of CE Data Resources. Help students master various cognitive tools item by item and use them skillfully, so that they can successfully carry out autonomous learning and collaborative learning, according to the application requirements of various learning tools needed by universities to experience the information-based learning environment of English. Freshmen are given a week to experience the English autonomous learning system when they first start school. This model satisfies current national CE teaching reform requirements, particularly those related to teaching model reform; for example, "the reform of teaching model should cause English teaching to develop toward individualization, independent of time and place, and active learning."

The CE data resource database is designed to help people share resources and manage large amounts of data. Teaching resources refer to the most basic materials used in network teaching, such as graphics and images, audio and video, multimedia lesson plans, network courseware, and a test question bank. Because the platform is primarily used to implement streaming media resources, the development of resources is primarily focused on streaming media teaching resources. This platform can help teachers make better use of their teaching materials.

To begin, a large number of scattered network resources, teaching film, and television materials are collated by schools for many years, and school-based curriculum resources are collected and stored as video files with chapters as the unit, and each video file is divided into $\mathrm{N}$ segments according to the teaching needs, and corresponding text streams and HTML page indexes are inserted at each segmentation point to find or index the corresponding tex. The platform is divided into three parts: a streaming media producer, a server, and a client. A model diagram of an application platform is shown in Figure 1. 


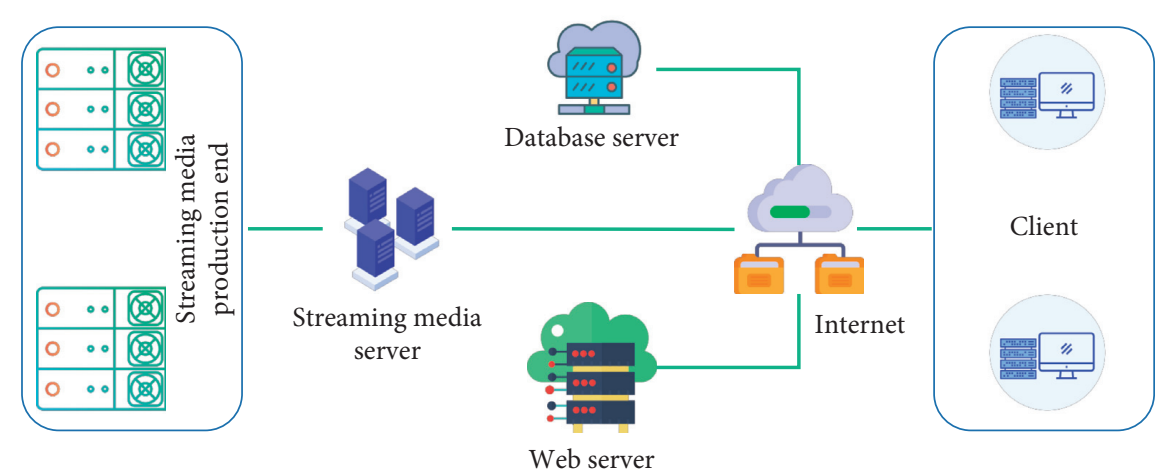

Figure 1: Model diagram of CE data resource pool.

The service interface subsystem provides external systems with interface services based on unified standards, such as the identity authentication interface and the resource metadata service interface. Metadata service interface includes metadata query, details browsing, behavior track, resource catalog, retrieval box docking, and other contents, which can meet the diversified resource application service needs of districts and schools. Figure 2 depicts the design of a specific functional structure.

The identity authentication interface and the metadata service class interface are both part of the service interface. Metadata service interface provides metadata query, details browsing, resource catalog, retrieval box docking, and other services. Identity authentication interface supports identity mutual trust among platforms based on joint authentication system. Metadata service interface provides metadata query, details browsing, resource catalog, retrieval box docking, and other services. A series of website security enhancements are being carried out in response to the characteristics of its Internet external service portal. The system patch is upgraded in time to strengthen the system security through vulnerability scanning and code auditing. Synchronous backup of the platform's core data is performed in the remote computer room to ensure that the remote computer room can carry web services if the main server room fails, and the digital resource sharing service platform's core services, such as web and exchange, can continue to operate.

Because each university offers a diverse range of disciplines and majors, the number of people who use it is naturally quite large, and each has its own set of needs, as well as different resources and materials. However, the web's educational resources system can meet such complex requirements, allowing for more open uploading, searching, and application of various teaching resources. With such a large resource pool, the system must meet specific storage capacity requirements, as well as stringent resource quality requirements. It is necessary to consider whether the resources are reliable and whether the system is in place.

Incorporate English teaching resources into the creation of digital English teaching materials, and gradually develop the school's own digital English teaching materials that are tailored to the needs of various grades, majors, and classes. Improve the usability of English textbooks and the rate at which they are used. From general English instruction to specialized English

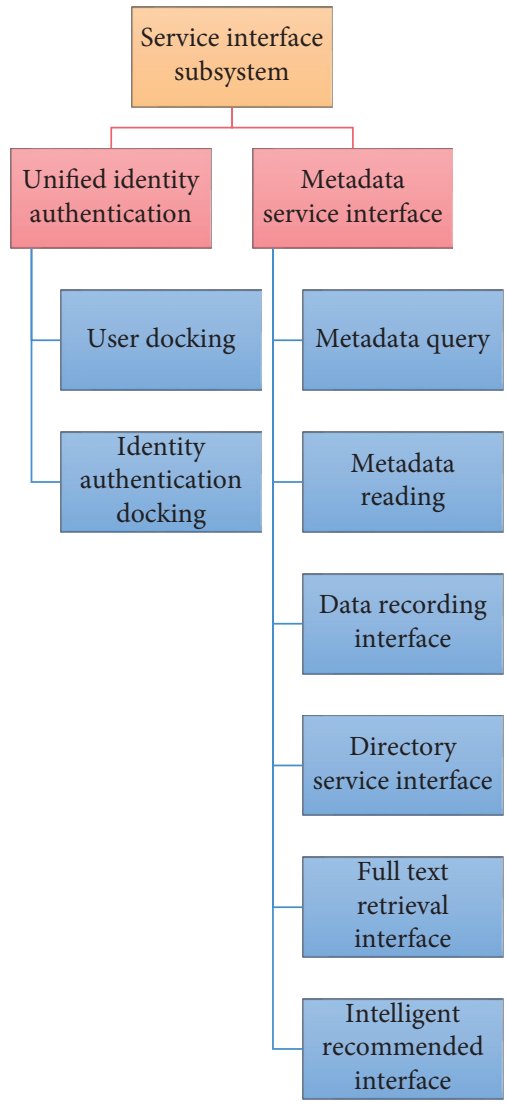

FIGURE 2: Service interface function structure.

instruction, we have got you covered. As a result, incorporating high-quality teaching resources is critical. English teachers should integrate existing teaching resources with advanced teaching equipment, recognize the added value of teaching information, and adhere to strict teaching objectives. Teachers should have a thorough understanding of the meaning of digital technology, be able to use multimedia technology effectively in the classroom, and organically integrate the purpose and content of English instruction.

3.2. Data Resource Optimization. Students are instructed, assisted, and encouraged to write "works" by their teachers. And the students are responsible for a large 
portion of the language input learning. Students learn in an autonomous learning center or over a local area network according to their own needs and levels, as determined by the teaching requirements and teachers' arrangements, embodying the principle of individualized instruction. Teachers can also recommend bibliographies and introduce network resources or Internet websites and assist students in developing the habit of self-study and increasing their self-assurance.

The units for task completion time, cost, and system load are all different. The fitness function is closely related to the comprehensive value of the weighted sum of these three indexes, so it is necessary to deal with the indexes so that there is no dimensional difference between them when designing the scheduling algorithm.

The actual value of the index and the evaluation value of the index are the two variables in a multiobjective comprehensive evaluation. There will be dimensional differences because each index has a different physical meaning. Resources in the cloud computing environment are dynamically heterogeneous, tasks are complex, and different types of tasks submitted by different users are also different. When designing GA, it is important to make assumptions about resource nodes and tasks and then base the algorithm's design on those assumptions, so that the algorithm can find a suitable allocation scheme for scheduling in a given environment.

Traditional network QoS (Quality of Service) usually refers to network speed, network reliability, and availability. QoS reflects consumers' satisfaction with the services provided. It is a comprehensive index. For example, QoS in the grid refers to the performance of a set of services, which determines the satisfaction of users with services. Research on QoS can be divided into three levels: application layer, system layer, and resource layer.

It expresses the objective function as the weighted sum of three-dimensional QoS objectives [23].

$$
M(x)=\omega_{1} * \text { time }+\omega_{2} * \text { cost }+\omega_{3} * \text { load },
$$

where $\omega_{1}+\omega_{2}+\omega_{3}=1, \omega_{1}, \omega_{2}, \omega_{3}$ refers to the weight coefficient of each index, time refers to the completion time, time refers to the cost, and load refers to the load.

Experimental tests show that the weight coefficient ratio of CPU (Central Processing Unit), memory, and bandwidth load is $4: 3: 15$ [48]. The penalty substitution iteration method is used to calculate the comprehensive load, and the specific formula is shown in formula (2).

$$
\text { load }=1-\prod_{k=1}^{3}\left(1-\operatorname{load}_{k}\right)^{\omega_{L k}}
$$

The overall goal of resource scheduling in this paper is to ensure system load while meeting users' QoS requirements, such as task time and cost. The three indicators are closely linked by comprehensive indicators. The smaller the values of these three indicators are, the more satisfactory the scheduling results can be for users, according to the problem analysis (Figure 3).
Suppose the population size is $m$, the fitness function value of individual $i$ is $f_{i}$, and the probability of $i$ being selected is flat $P_{i}$.

$$
P_{i}=\frac{f_{i}}{\sum_{i=1}^{n} f} .
$$

$P_{i}$ represents the proportion of the fitness of individual $i$ in the sum of all individual fitness in the population. The greater the fitness value of an individual, the greater the chance of being selected.

The execution time of the update transaction job is not fixed due to various factors that may affect the actual update of the transaction, but it often changes dynamically with the influence of the actual running environment of the system at the time, and this change is sometimes very significant and substantial. The purpose of this paper is to improve and supplement the shortcomings of the DS-FP (DeferringScheduling Fixed-Priority) method in the application of software/fixed real-time systems. The calculation time of updating transaction jobs obeys a certain probability density function distribution [24] after using the probability statistics method to design the system, ensuring that the results of job scheduling and transaction execution meet the system's performance requirements.

In DS-PS method, for $i<j$, suppose $T_{i}<T_{j}$, when any job $J_{i, j}$ of $T_{i}$ is ready; the worst case is that all jobs with priority not lower than $J_{i, j}$ are also ready at the same time. If the sampling time $r_{i, j+1}$ of the next job $J_{i, j+1}$ of transaction job $J_{i, j}$ is delayed to $r_{i, j+1}$, then:

$$
r_{i, j+1}^{\prime}=d_{i, j+1}-R_{i, j+1}\left(r_{i, j+1}^{\prime}, d_{i, j+1}\right),
$$

where $R_{i, j+1}\left(r_{i, j+1}^{\prime}, d_{i, j+1}\right)$ is the response time of job $J_{i, j+1}$ in $\left[r_{i, j+1}^{\prime}, d_{i, j+1}\right)$. In this case, $J_{i, j+1}$ is allowed to have the following number of jobs ready at most in $T_{i}$ within $\left[r_{i, j+1}^{\prime}, d_{i, j+1}\right)$ time period after sampling:

$$
h p_{i}\left(d_{i, j+1}-r_{i, j+1}^{\prime}\right)=\sum_{i^{\prime}=1}^{i-1}\left[\frac{d_{i, j+1}-r_{i, j+1}^{\prime}}{P_{i^{\prime}}}\right] .
$$

Within $\left[r_{i, j+1}^{\prime}, d_{i, j+1}\right)$ time, the execution time of all jobs with high priority $J_{i, j+1}$ in the system:

$$
\Theta_{i}\left(r_{i, j+1}^{\prime}, d_{i, j+1}\right)=\sum_{i^{\prime}=1}^{m} \sum_{j=1}^{h p_{i^{\prime}}(t)} C_{i^{\prime}, j} .
$$

The formula (4) is as follows:

$$
R_{i, j+1}\left(r_{, j+1}^{\prime}, d_{i, j+1}\right)=\sum_{i^{\prime}=1}^{m} \sum_{j=1}^{h p_{i^{\prime}}(t)} C_{i^{\prime}, j}+C_{i, j+1} .
$$

Therefore, DS-PS algorithm must obtain the delayed sampling time and absolute deadline of all high priority jobs before calculating $\Theta\left(r_{i, j+1}^{\prime}, d_{i, j+1}\right)$.

3.3. The Orientation Change of CE Teachers. The transformation and orientation of CE teachers' functions have become unavoidable, according to research on the 


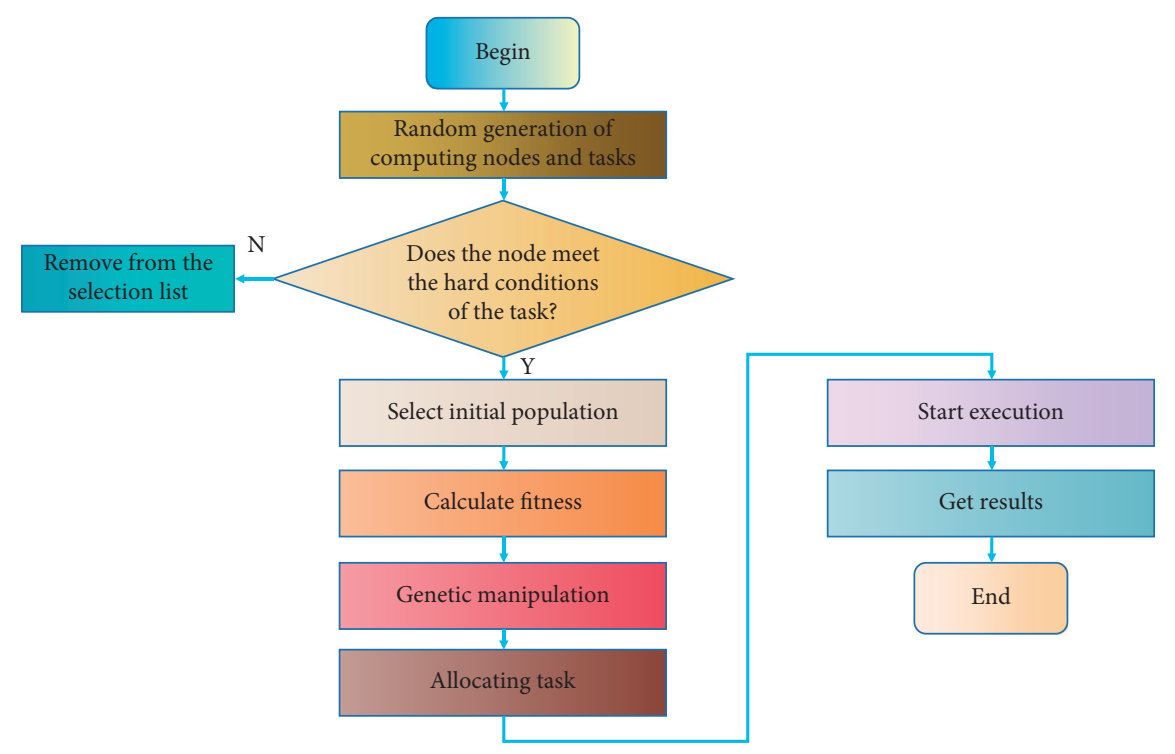

FIgURE 3: System diagram of resource evaluation model.

development status of $\mathrm{CE}$ teachers supported by digital technology.

Students actively collect and analyze relevant information and materials under the guidance of teachers, using multimedia and the Internet, and try to relate what they learn in the current learning content to what they already know. Effectively construct the meaning of the learned knowledge through the guidance of teachers and communication, discussion, and cooperation with learning partners, in order to improve our ability of information collection, information analysis, information processing, information innovation, and information expression, as well as cultivate our spirit of cooperation and innovation.

English teachers should use digital technology to create an active learning environment for their students. To achieve the best effect of English teaching, teachers should actively build a bond of trust in English teaching, release students' learning pressure, and eliminate their inferiority complex through two-way communication between teachers and students [7].

Teachers and students can use the Internet to ask and answer questions. This necessitates that teachers and students understand each other's teaching effect and that both sides work together to efficiently organize the teaching process in order to maximize the teaching effect. At the same time, students must understand their own learning effect after a period of study. The teacher's role at this time is also that of an evaluator, pointing out the students' accomplishments and advantages while also offering suggestions and guidance for their problems. Students will have a more accurate assessment of their learning situation if they are evaluated on time and in a reasonable manner. They will also be better prepared to learn in the future if they are evaluated on time and in a reasonable manner. The intrinsic motivation to motivate students to be positive is positive evaluation. Teachers should abandon traditional evaluation methods that primarily focused on test scores in favor of testing and evaluating students' learning performance and effect [9]. To ensure fairness and rationality in evaluation, teachers should conduct comprehensive evaluations on students, provide patient guidance and inspiration, and pay attention to the combination of personalized and process evaluations.

\section{Results Analysis and Discussion}

The more hands-on activities they participate in in the classroom, the better their hands-on ability, creativity, and sense of gain will improve. Teachers are proud of what they have accomplished. Under the information environment, there are two parts to the construction of CE digital teaching resources and teaching materials: English digital teaching resources construction and teaching materials construction.

In this experiment, the data involved are the attribute and number of cloud computing tasks, the attribute and number of resource nodes, the user's demand weight for time, cost, and load, the population size of GA, etc. Figure 4 shows the attribute values of 10 randomly generated tasks.

As an educator, even in the environment of students' autonomous learning, consider teaching issues from the perspective of pedagogy, and use nontechnical factors such as our emotions to urge students to learn better. At the same time, emphasize the psychological changes of students in the learning process, and solve their psychological problems in time. In addition, English teachers should strengthen their own research, be a researcher, keep up with the development of various ideological trends and new technologies, and apply the latest theories and technologies to teaching.

The results of simulation experiments show that the algorithm proposed in this paper is capable of selecting appropriate scheduling nodes for tasks based on users' needs and generating a satisfactory allocation scheme. The algorithm in [15] is compared to the algorithm in [19] in three ways: task completion time, cost, and the value of the 


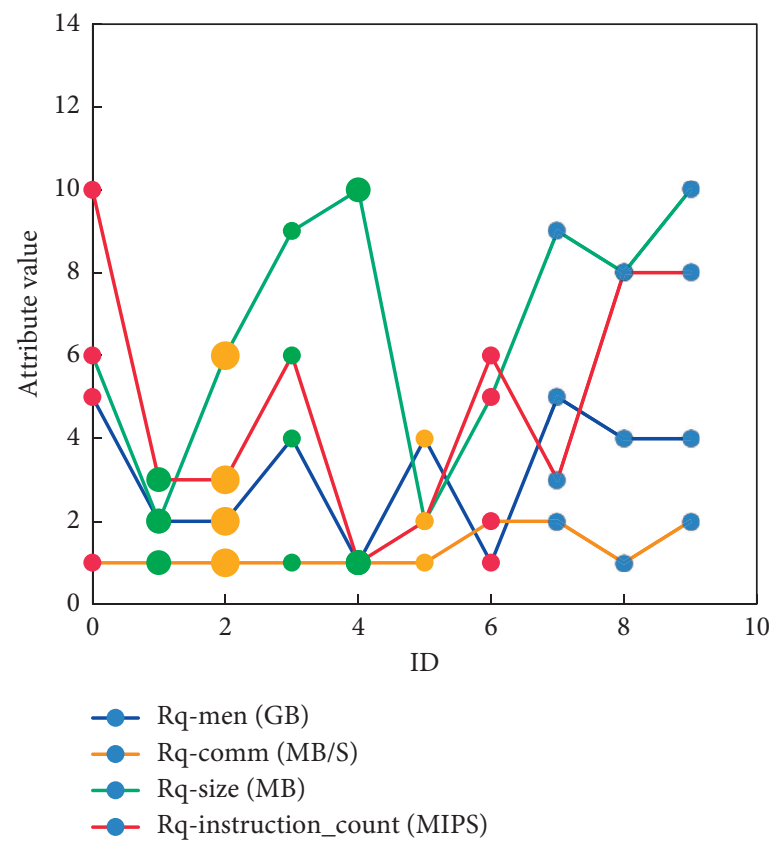

FIGURE 4: Task attribute value.

comprehensive objective function. The results shown in Figure 5 are the ones that were obtained.

Ninety percent of users of cloud computing want to get satisfactory service at the lowest possible cost. Analyzing from the constraint index of cost, the cost of the three algorithms will be higher and higher, but the cost of the algorithm in this paper is less than that of the algorithms in $[15,19]$.

In classroom teaching, teachers communicate with students mainly by asking questions. The learning process adopts the static teaching method, and students' learning thinking is limited, so they cannot learn independently, and it is difficult to communicate and exchange with English knowledge.

English teachers must improve their classroom knowledge, teaching activity design, student psychology, and teaching resource development, among other things. The teacher's role is similar to that of a traditional teacher in this regard. Teachers should plan and organize an entire stage of the teaching process, not just a single class, that is, in a macrosense, the organizer. This demonstrates that teachers should plan and organize the selection of students' learning materials, the formulation of students' learning objectives, the organization of the learning process, and the evaluation of learning effect based on the students' current situation. Figure 6 shows that as the number of tasks grows, the time required by the three scheduling algorithms grows. However, the algorithm in [19] takes less time to complete in total than the algorithm in this paper.

The objective function values of the two algorithms are very similar when the number of tasks is less than 40 , but when the number of tasks exceeds 40 , the objective function values obtained by the algorithm proposed in this paper are less than those obtained by the algorithms in [15] and [19].
As a result shown in Figure 7, in terms of overall performance, this algorithm outperforms the other two algorithms.

The system's load is an important consideration when allocating resources. Users can make the algorithm choose the best computing node for the task based on their own load weight coefficient setting through simulation experiments. The worth of a load will rise as well. Because the weight coefficients are set differently, the load values of the three algorithms will differ. The load calculation of the algorithm in [15], on the other hand, is based on a formula that ignores the needs of users. As a result, from a broad perspective, this algorithm can better meet various QoS requirements.

As shown in Figure 8, the real-time data object can guarantee the timing consistency requirement in this update transaction scheduling process, ensuring the system's overall performance. The first two methods can also guarantee 100 percent of the service quality requested by users and even improve the execution service quality above the service quality requested by users. When deterministic scheduling methods, such as those in [21] and [23], are applied in the software/fixed real-time system, it is shown that our previous strategy of reducing the schedulability of the system to ensure the correctness of the system performance is really harsh or pessimistic.

The network's all-around communication between teachers and students, as well as students and students, has reduced the psychological distance between them and increased the opportunities and scope of communication between them. Teachers can better understand the doubts, difficulties, and main problems that students face in learning by using statistical analysis of the types, times, and numbers of students' questions, which provides a realistic and effective way for personalized teaching. The CPU resource 


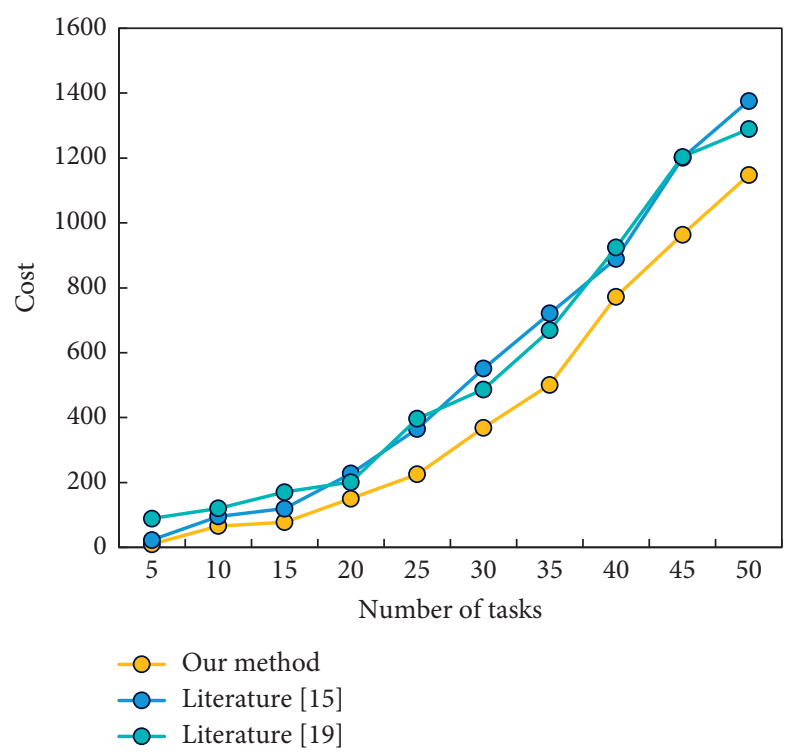

Figure 5: Cost comparison chart.

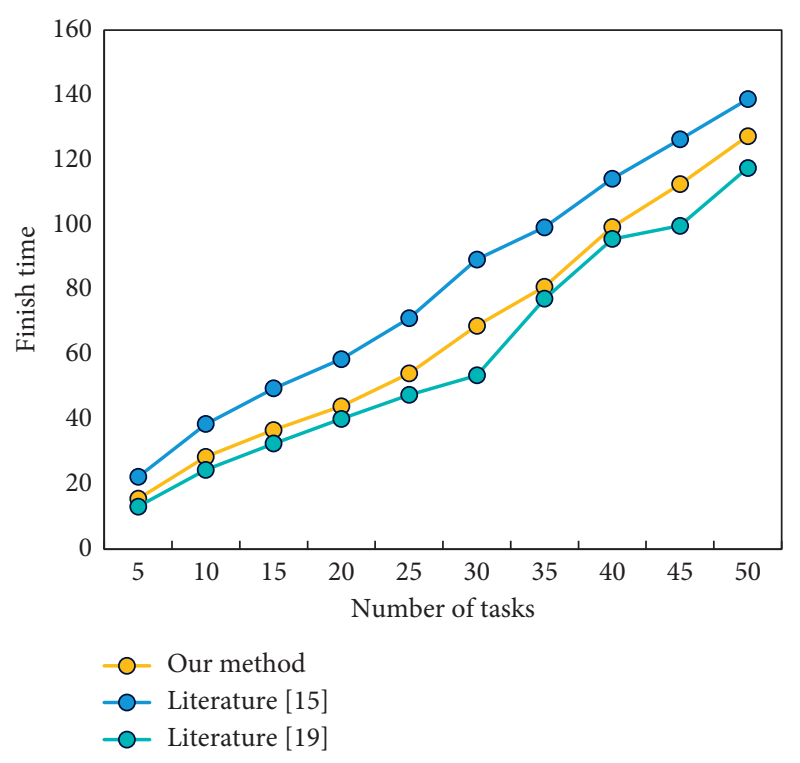

FIGURE 6: Comparison chart of completion time.

utilization rate of the three methods increases monotonically with the number of scheduled update transactions, as shown in Figure 9.

The main goal of DS-PS method is verified and realized (that is, while trying to delay the cycle of transaction operation, the calculation time of the operation is less than that in the worst case, thus reducing the utilization rate of CPU resources in the system).

Students-centered, teaching-centered programs improve students' overall performance, help them adapt to job demands, and help them better serve society, improving unified planning and design in the resource database building process, as well as integrating the resource application level. Teachers can use the platform of teaching

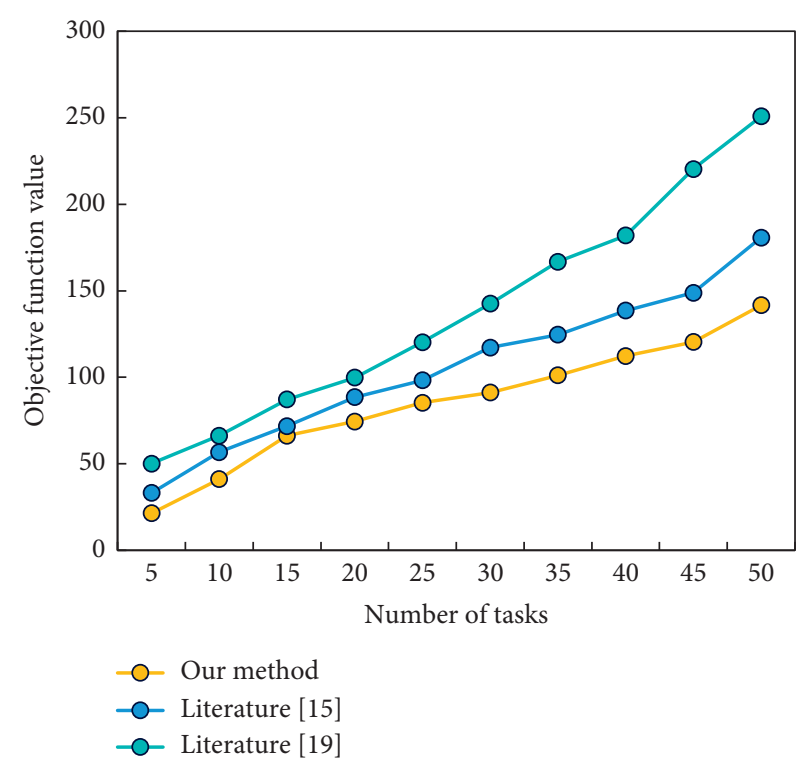

Figure 7: Comparison chart of objective function values.

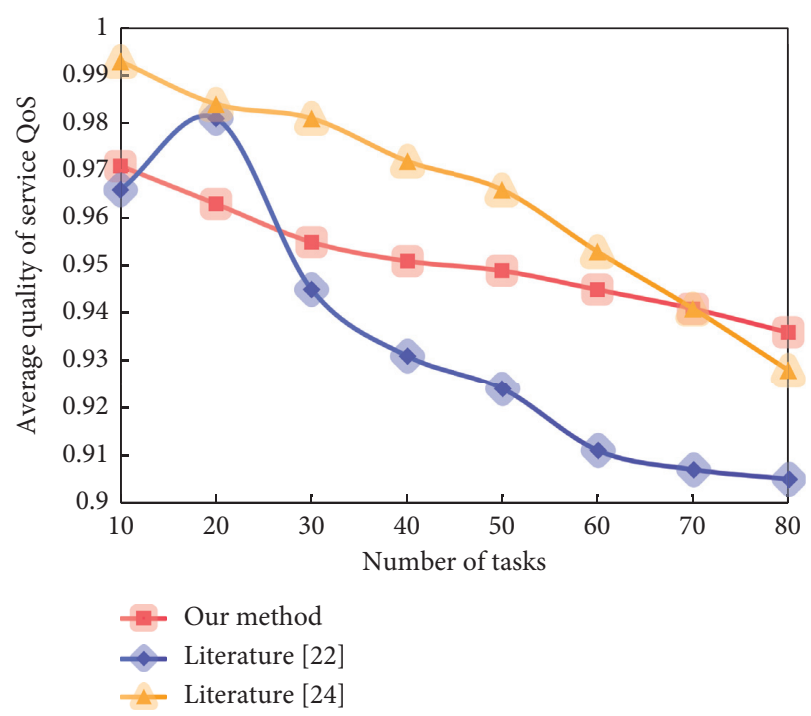

FIgURE 8: Comparison of the relationship between the average QoS of arrival of three methods and the number of transactions executed.

resource pool to select the best teaching resources, design the most effective teaching process, achieve the best classroom teaching effect, meet the needs of students, and complete the teaching objectives.

The CPU resource utilization ratio of the method in [21] and the method in [23] when executing the scheduling update transaction under the condition that the QoS requested by users is different remains equal, as shown in Figure 10, because the system will still strictly require that the update transaction be executed to provide the service quality guarantee, even if the service quality requested by users is processed by these two methods. 


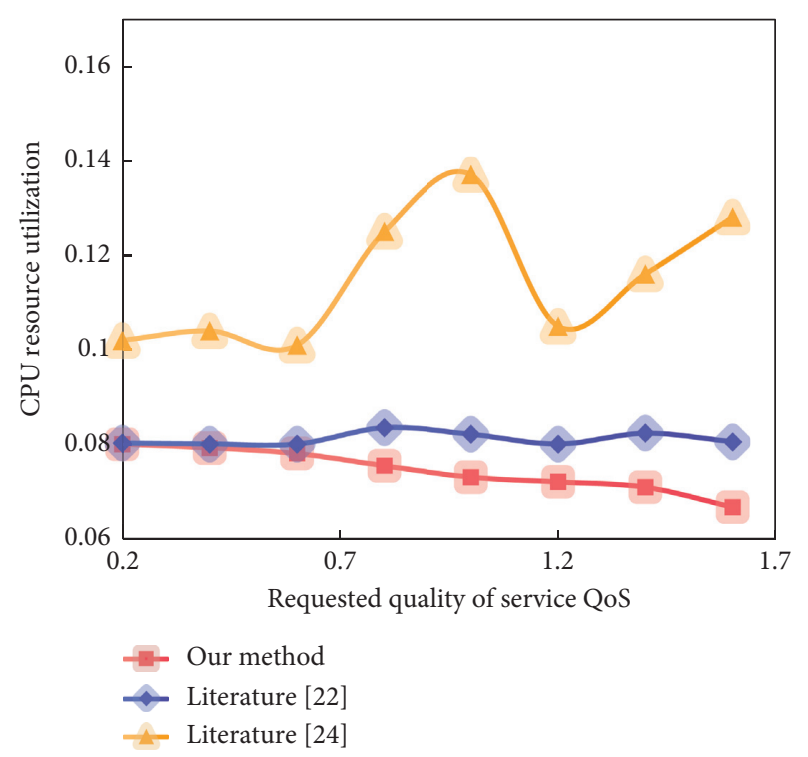

FIGURE 9: Comparison of the relationship between the number of transactions scheduled by three methods and the utilization rate of CPU resources.

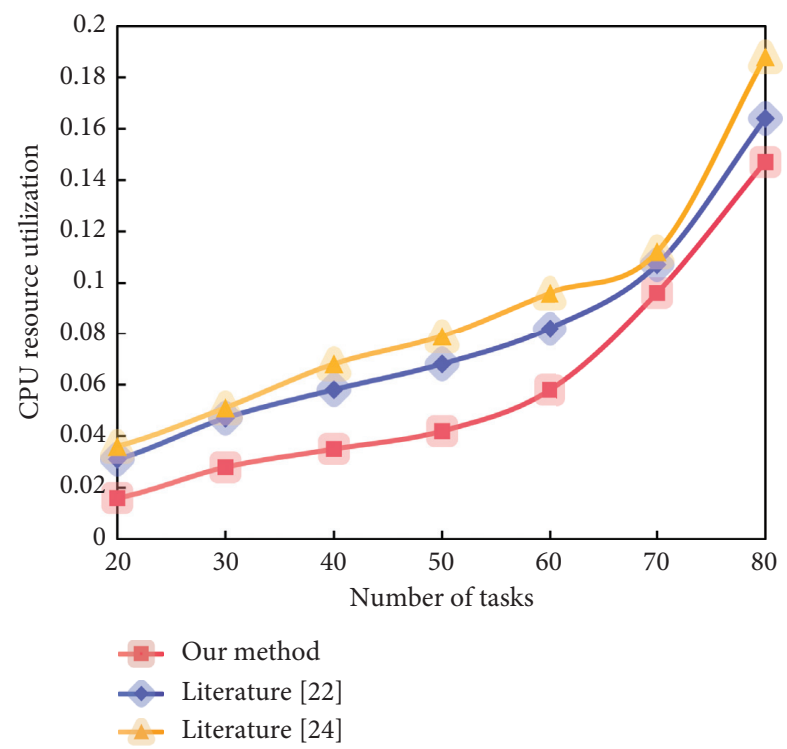

Figure 10: Comparison of three methods in relation between requested QoS and CPU resource utilization.

This also demonstrates that the DS-PS method can lower the quality of service of requests in exchange for increased system schedulability, making the DS-PS method more flexible in scheduling transaction job update operations and thus improving system performance.

Update resource management in the resource library on a regular basis, and improve resource construction management and application. Schools should use corresponding incentive mechanisms to ensure the long-term and dynamic construction process of resource pools in order to study the long-term mechanism of resource standardization and resource pool construction, acting as planners, organizers, guides, facilitators, designers, managers, supervisors, monitors, collaborators, and evaluators in the classroom to mediate students' learning process from teaching content, teaching methods, task setting and implementation, and other factors. This necessitates that teachers possess certain qualities, including knowledge of pedagogy, psychology, and other related topics, as well as the ability to use modern information technology, such as multimedia and network technology.

\section{Conclusion}

This research presents a relatively comprehensive construction scheme for a CE data resource base based on a web database, which allows users to upload, edit, download, read, and use teaching multimedia resources. GA is designed to solve the resource scheduling problem, and the objective function is obtained by integrating the three indexes of task completion time, cost, and load balance. The process of algorithm execution, preconditions, and assumptions, the unique environment of cloud computing, and the strategy selection of three types of genetic operations are all examined in this paper. In a cloud computing environment, a resource scheduling optimization strategy based on QoS constraints and GA is proposed. CE data resource pool can further integrate CE high-quality resources to achieve balanced supply, which has significant social benefits and plays an important role in expanding the coverage of CE highquality education resources, promoting education equity, and improving the quality of school education and teaching.

\section{Data Availability}

The data used to support the findings of this study are included within the article.

\section{Conflicts of Interest}

The author does not have any possible conflicts of interest.

\section{Acknowledgments}

This study was supported by Practice and Reflection on the Process Evaluation of English Teaching Reform in Higher Vocational Colleges based on "Chao Xing" platform (2020011).

\section{References}

[1] G. Wang, "On the application of cooperative learning in college English teaching," International Education Studies, vol. 13 , no. 6 , p. $62,2020$.

[2] P. Feasibility, "Analysis of CE teaching based on the Mastery Flipped mode," Journal of Hubei university of Education, vol. 036, no. 3, pp. 83-86, 2019.

[3] R. T. Borst, P. M. Kruyen, and C. J. Lako, "Exploring the job demands-resources model of work Engagement in Government: bringing in a psychological perspective," Review of Public Personnel Administration, vol. 39, no. 3, pp. 372-397, 2019.

[4] D. Grace, S. Weaven, K. Bodey, M. Ross, and K. Weaven, "Putting student evaluations into perspective: the course 
experience quality and satisfaction model (CEQS)," Studies In Educational Evaluation, vol. 38, no. 2, pp. 35-43, 2012.

[5] J. Tian, "Optimization of Embedded mobile Teaching Model Based on Network Streaming media Technology," Complexity, vol. 2021, Article ID 3449338, 2021.

[6] L. Zhijuan and Z. Jiaming, "Application of streaming media technology in nursing teaching," in Proceedings of the IEEE International Symposium on IT in Medicine and Education, vol. 1, pp. 70-74, IEEE, Guangzhou, China, December 2011.

[7] T. Kjærgaad, "Rhizomes and plateaus: a study of digital communities of practice in University CE Teaching," Afore, vol. 55, no. 1, pp. 35-45, 2017.

[8] G. Wei-xing, "Application of learning design into the cultivation of Multiliteracies: a case study of CE teaching practice at," Soochow University, vol. 4, pp. 125-143, 2021.

[9] X. Feng, "Mindset rather than technology is what really Matters in Flipped classroom in CE teaching:an organization development perspective," A summary of educational theories, vol. 3 , no. 1, p. 6, 2020.

[10] L. Wang, "Analysis of CE teaching in application oriented colleges and universities based on the effect oriented concept," Contemporary educational research, vol. 5, no. 2, p. 3, 2021.

[11] H. Jiang and Q. Xu, "Multimedia CE teaching based upon the behaviorism theory," Journal of Educational Institute of Jilin Province, vol. 6, pp. 76-77, 2021.

[12] Y. Feng, “The Implications of Krashen's second language Acquisition theory on CE teaching," Overseas English, vol. 1, no. 1, p. 2, 2020.

[13] Q. X. Sun, "The application of Flipped classroom mode in CE teaching based on MOOCs," Literature \& Art Studies: English Version, vol. 10, no. 6, p. 4, 2020.

[14] S. Zhang, "Challenges facing the CE teaching of Preschool education," Business (Economic Management), vol. 6, no. 6, p. $1,2020$.

[15] P. Györgyi, "A PTAS for a resource scheduling problem with arbitrary number of parallel machines," Operations Research Letters, vol. 45, no. 6, pp. 604-609, 2017.

[16] T. Liu, Y. Zhang, Y. Zhu, T. Weiqin, and Y. Yuanyuan, "Online Computation Offloading and resource scheduling in mobile Edge computing," IEEE Internet of Things Journal, no. 99, 2021.

[17] J. S. Kumar, M. A. Zaveri, and M. Choksi, “Task based resource scheduling in IoT environment for Disaster management," Procedia Computer Science, vol. 115, pp. 846-852, 2017.

[18] F. Al-Tam, N. Correia, and J. Rodriguez, "Learn to Schedule (LEASCH): a Deep reinforcement learning approach for radio resource scheduling in the 5G MAC layer," IEEE Access, vol. 8, no. 99, 2020.

[19] A. Arif, Z. Wang, C. Chen, and J. Wang, "Repair and resource scheduling in Unbalanced distribution systems using Neighborhood Search," IEEE Transactions on Smart Grid, vol. 11, no. 99, 2019.

[20] C. Gza, Z. Hao, B. Yla, L. Yanling, and X. Lei, "5G networkoriented hierarchical distributed cloud computing system resource optimization scheduling and allocation," Computer Communications, vol. 164, pp. 88-99, 2020.

[21] M. Mohseni, S. A. Banani, A. W. Eckford, and R. S. Adve, "Scheduling for VoLTE: resource allocation optimization and Low-Complexity algorithms," IEEE Transactions on Wireless Communications, vol. 18, no. 3, pp. 1534-1547, 2019.

[22] G. Xu, Y. Bai, Q. Pan, Q. Huang, and Y. Yang, "Data verification tasks scheduling based on dynamic resource allocation in mobile big data storage," Computer Networks, vol. 126, pp. 246-255, 2017.

[23] A. Mhm, A. Molin, and D. Tolić, "Error-dependent data scheduling in resource-aware multi-loop networked control systems - ScienceDirect," Automatica, vol. 81, pp. 209-216, 2017.

[24] J. Wan, B. Yin, D. Li, A. Celesti, F. Tao, and Q. Hua, "An Ontology-based resource Reconfiguration method for Manufacturing Cyber-physical systems," IEEE, vol. 23, no. 6, pp. 2537-2546, 2018. 